\title{
Supplementary Information \\ Feature Ranking and Modeling of Mineral Effects on Reservoir Rock Surface Chemistry Using Smart Algorithms
}

Isah Mohammed - (Ph.D. Student)

Petroleum Engineering Department, College of Petroleum Engineering and Geosciences, King Fahd University of Petroleum and Minerals, Dhahran 31261, Saudi Arabia.

Email: g201806900@kfupm.edu.sa

*Dhafer Al Shehri - (Chairman)

Petroleum Engineering Department, College of Petroleum Engineering and Geosciences, King Fahd University of Petroleum and Minerals, Dhahran 31261, Saudi Arabia.

Phone: +966 (013) 860-2536;

Email: alshehrida@kfupm.edu.sa.

* Corresponding author email:alshehrida@kfupm.edu.sa.

*Mohamed Mahmoud - (Professor)

Petroleum Engineering Department, College of Petroleum Engineering and Geosciences, King Fahd University of Petroleum and Minerals, Dhahran 31261, Saudi Arabia.

Phone: +966 (013) 860-2524;

mmahmoud@kfupm.edu.sa.

* Corresponding author email: mmahmoud@kfupm.edu.sa

Muhammad Shahzad Kamal - (Research engineer II)

Center for Integrative Petroleum Research (CIPR),

King Fahd University of Petroleum and Minerals, Dhahran 31261, Saudi Arabia.

Email: shahzadmalik@kfupm.edu.sa

Olalekan Saheed Alade - (Research Engineer I)

Center for Integrative Petroleum Research (CIPR),

King Fahd University of Petroleum and Minerals, Dhahran 31261, Saudi Arabia.

Email: olalekan.alade@kfupm.edu.sa 


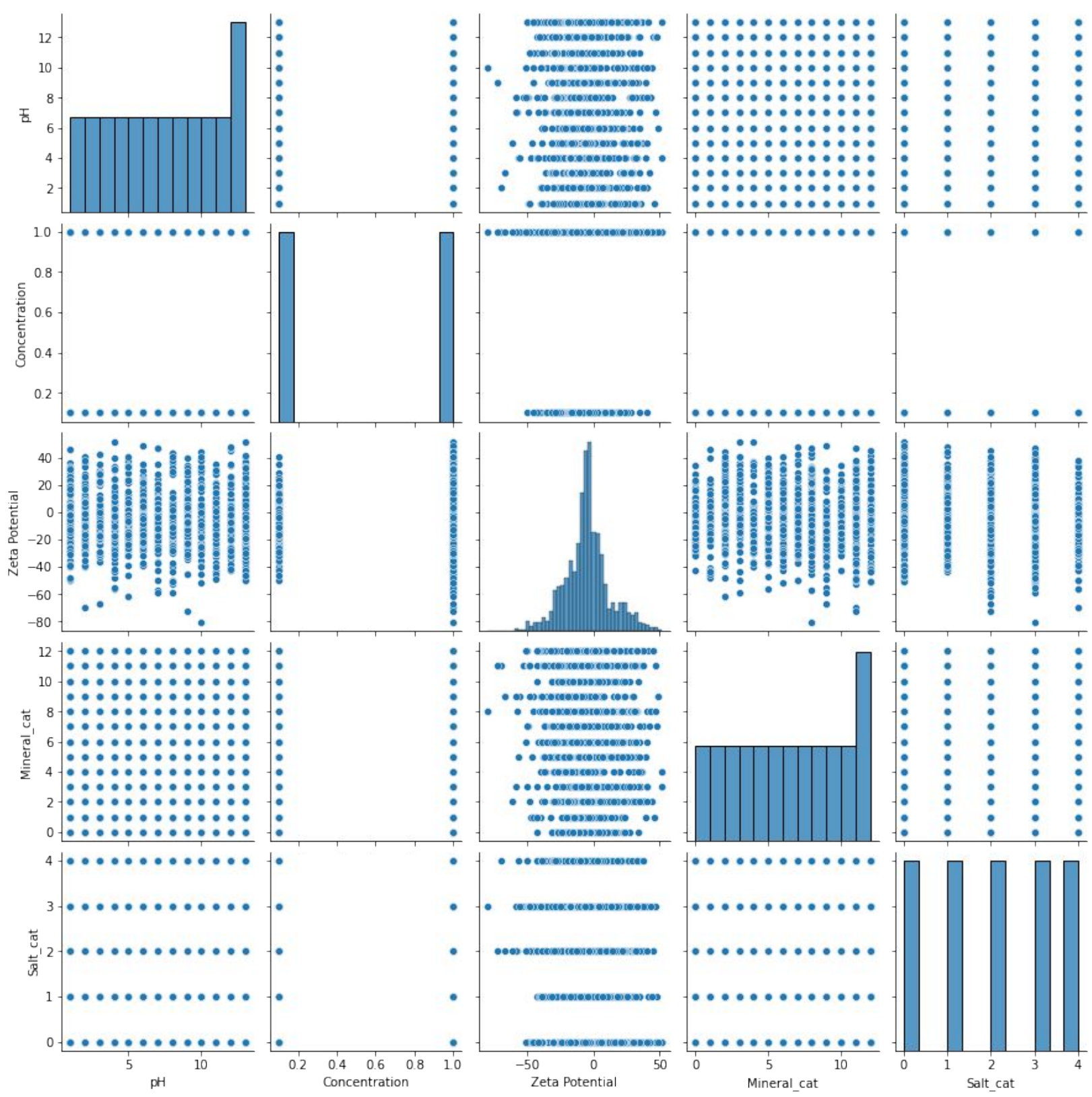

Figure S1: Pair plot showing data distribution and correlation 
(A) SVR
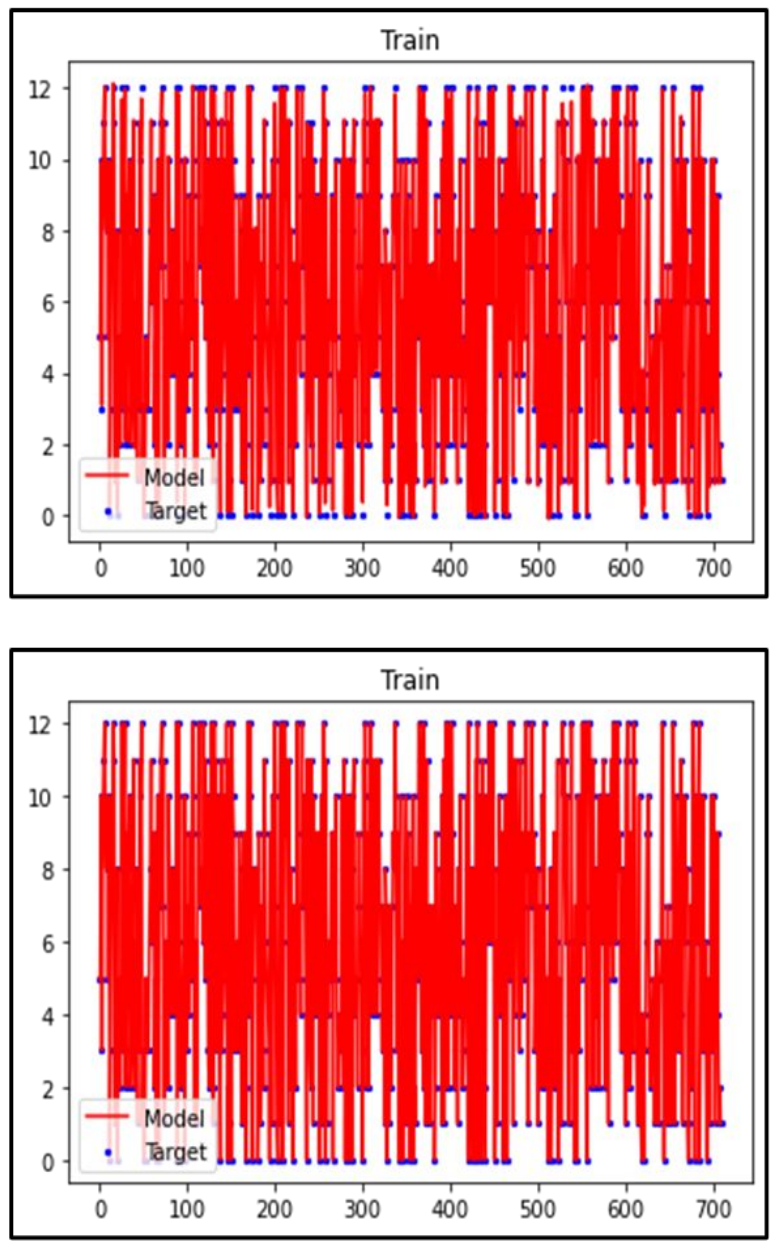

Figure S2: Model performance for (a) SVR and (b) ADA

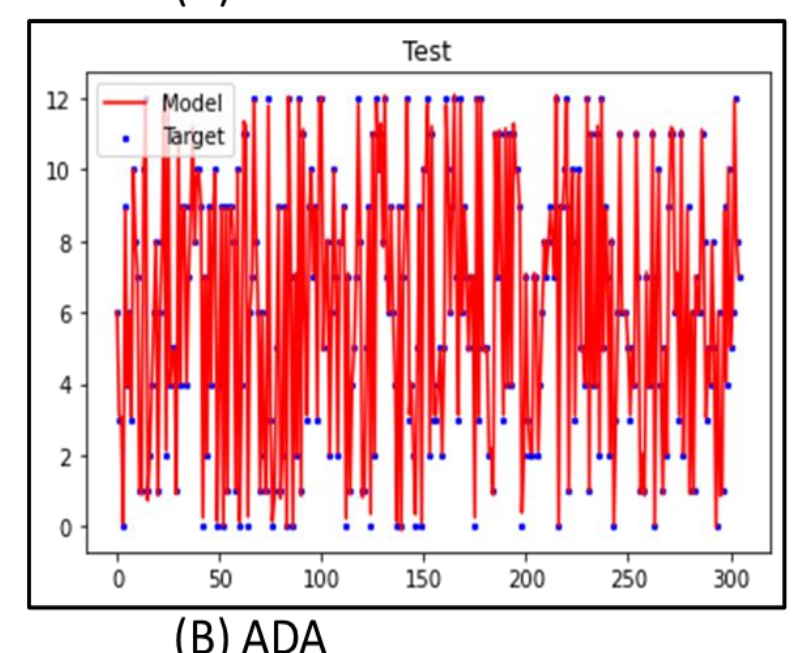

(B) ADA

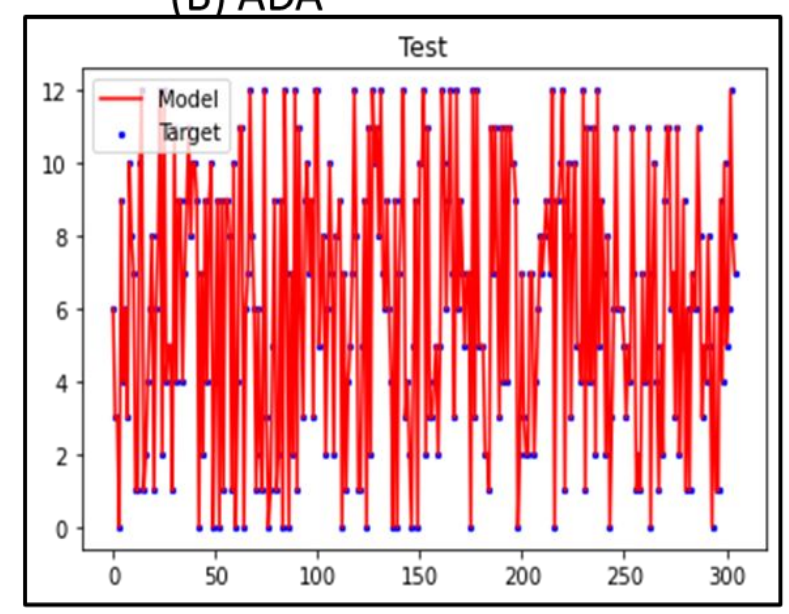

Validation
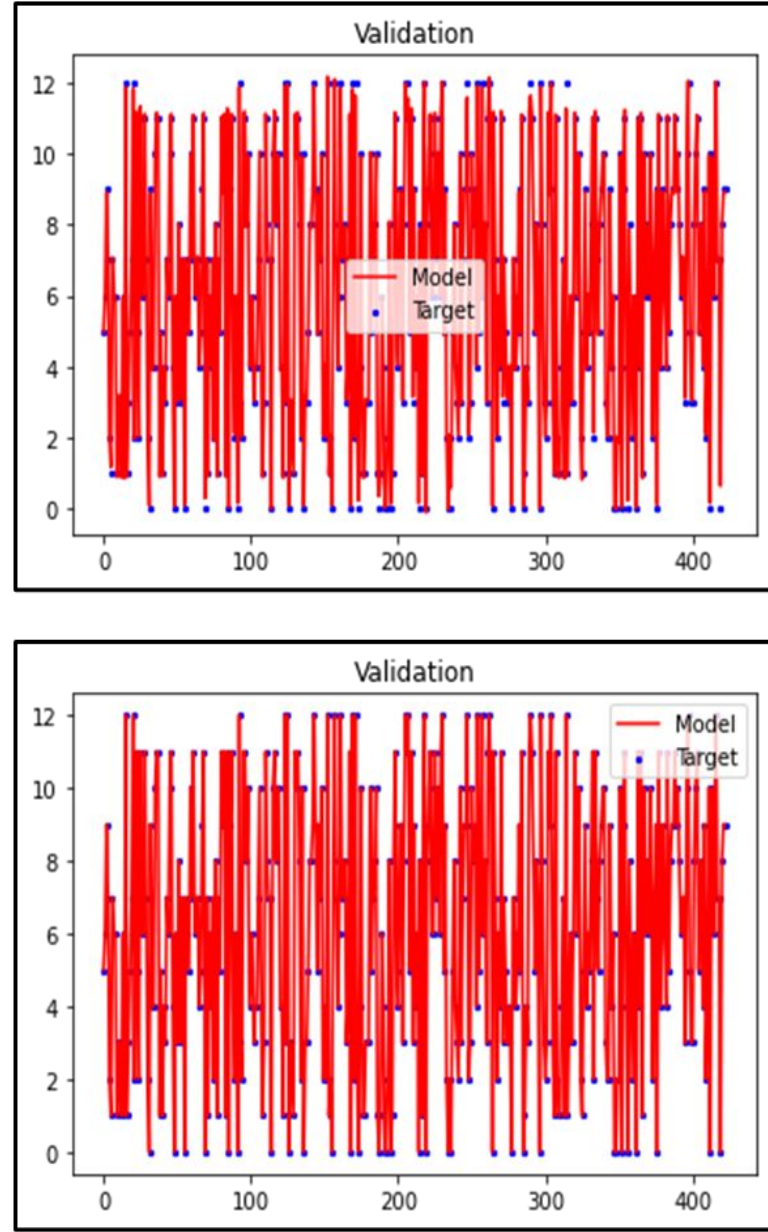

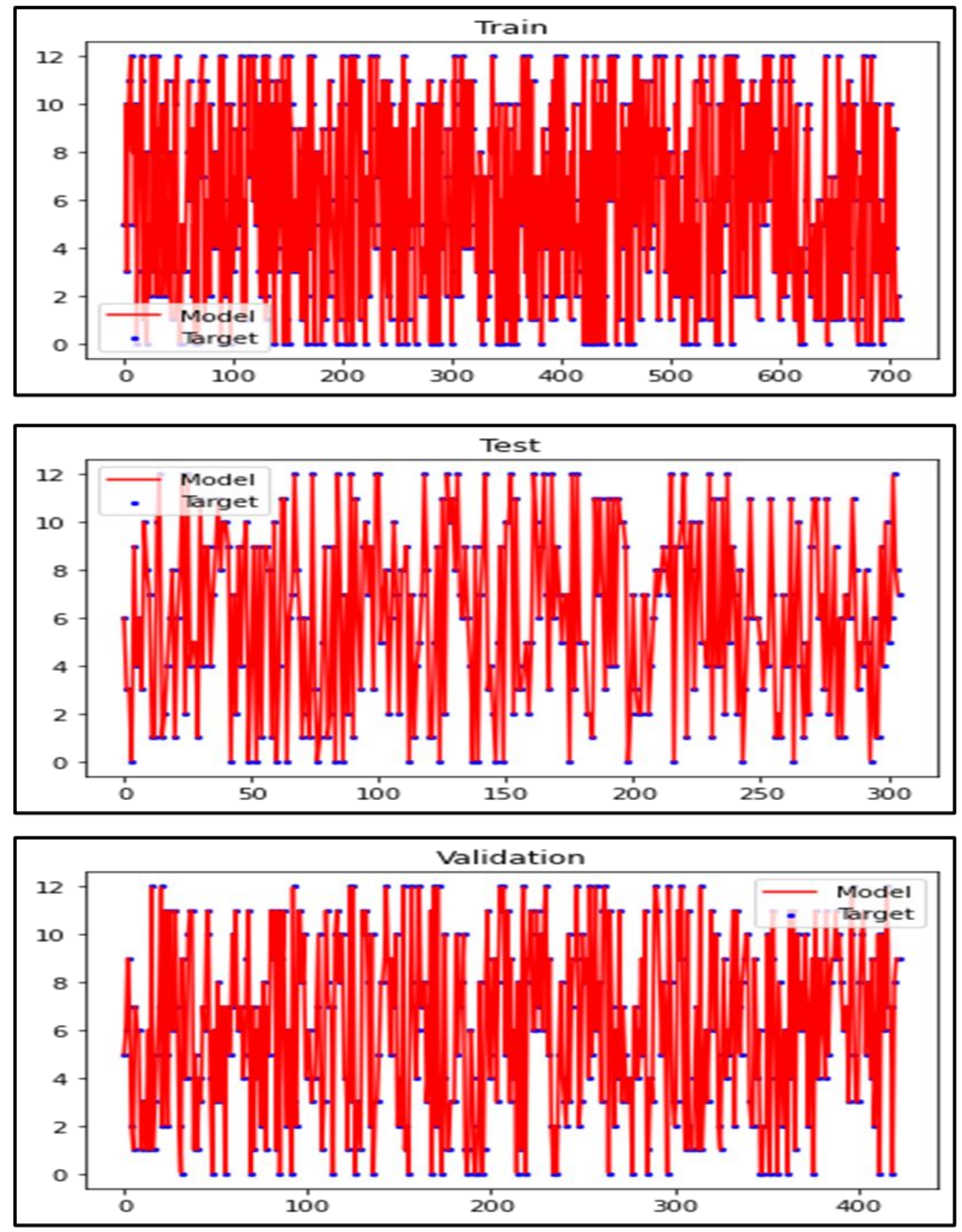

Figure S3: Model performance for GDT 
